\title{
Fast Association Process (FAP) of Beacon Enabled for IEEE 802.15.4 in Strong Mobility
}

\author{
Muhammad Javed, Kartinah Zen and Halikul Bin Lenando \\ Faculty of Computer Sciences and Information Technologies, \\ University Malaysia Sarawak Kuching, Malaysia \\ javed.sikandry@gmail.com, \{Kartinah and cool\}@fit.unimas.my
}

\author{
Hushairi Zen \\ Faculty of Engineering, \\ University Malaysia Sarawak Kuching, Malaysia \\ zhushair@feng.unimas.my
}

\begin{abstract}
In strong mobility the mobile node association with a coordinator (static or mobile) is an important part of IEEE802.15.4 protocol. This research analyzes the mobile node association attempt process flows in detail. This research also proposes an enhanced association procedure names Fast Association Process (FAP) in strong mobility. FAP is introduced with new Association_Data request MAC command that increases the association period and provides fast association process in strong mobility. It reduces the redundant service primitives, avoid collision and decrease association attempt process delay. Comparing FAP with the original IEEE802.15.4 protocol, the number of association service primitives in FAP is $67 \%$ less than the original protocol, and the simulation results show that the association attempt time decreases $75 \%$. FAP will get fast association attempt as the number of mobile nodes increased and nodes having strong mobility. It can be widely used in mobile wireless sensor network application.
\end{abstract}

Keywords-fast association process; IEEE802.15.4; strong mobility; beacon enabled

\section{INTRODUCTION}

The IEEE 802.15.4 is a member of the IEEE802 standards, but all the characteristics of other IEEE802 standards are not included for this low-rate, low-complexity, and low-cost and low-power wireless connectivity among inexpensive nodes in wireless personal area networks (LR-WPAN) [1]. The aim of this standard was to able the users who had low skill or interest in the radio technology or communication protocol skills to effectively use and benefit from radio based standards. These features determine its huge potential in industry, floods, earthquake, battlefield surveillance, home automation, mobile electronic system, agriculture, intelligent transportation system, medical sensors and so on. The task of this group is to maintain standard for Physical (PHY) layer and Media Access Control (MAC) layer. The higher layers are handled by ZigBee alliance which was founded in August 2002. The applications supported by ZigBee include home automation, remote control and monitor and health care [2].

This low-rate wireless personal area network (LR-WPAN) standard defines two types of physical devices. The Full Function Device (FFD) can communicate with Reduced
Function Device (RFD) or other FFDs. An RFD can communicate with only FFD. An RFD may only associate with a single FFD at a time. The RFD can be implemented using minimum resources and memory space [1].

There are three logical devices: the PAN coordinator, the coordinator, and the End Devices. The PAN coordinator is built from an FFD, and responsible for establishing a new PAN network. The PAN coordinator has overall information of the entire PAN network. The coordinator is built from the same FFD. Its primary task in PAN network is to route packets and associate end devices. The last logical device is End Device. End Device is built from an RFD. It handles only communication and data transfer [1]. Depending on application requirements, the LR-WPAN can establish two topologies: The star topology or the peer-to-peer topology. In star topology (as shown in Fig. 1), every node could only communicate with coordinator or PAN coordinator.

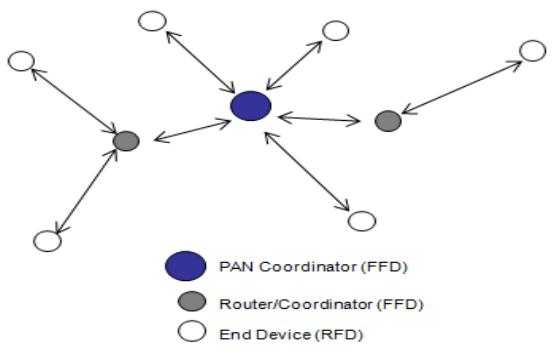

Fig. 1: Star topology

The IEEE Std $802.15 .4^{\mathrm{TM}}-2003$ is not a static-even now, there are amendments and additions, explanations and generalizations being debated that are improving the service of the standards. Second version released in 2006 as IEEE Std $802.15 .4^{\mathrm{TM}}-2006$ and recently published version is IEEE Std $802.15 .4^{\mathrm{TM}}-2011$. 